\title{
Erratum
}

\section{Erratum to "Influence of Stratospheric Intrusion on the Surface Ozone Levels in India"}

\author{
Nandita D. Ganguly \\ Department of Physics, St. Xavier's College, Ahmedabad 380009, India \\ Correspondence should be addressed to Nandita D. Ganguly, ganguly.nandita@gmail.com \\ Received 9 August 2012; Accepted 29 August 2012 \\ Copyright (C) 2012 Nandita D. Ganguly. This is an open access article distributed under the Creative Commons Attribution \\ License, which permits unrestricted use, distribution, and reproduction in any medium, provided the original work is properly \\ cited.
}

(1) "The PV value was $>1.6$ and $\mathrm{RH}$ at the tropopause were low (20\%), indicating the presence of air masses having stratospheric origin," in page 3, column 2, 4th last line, should be changed to "The PV value at the tropopause was $>1.6$ and $\mathrm{RH}$ at $300 \mathrm{hPa}$ pressure level was low (20\%), indicating the presence of air masses having stratospheric origin."

(2) In pages 4-5 in Figures 4(a)-4(i), (a, b, c, d, e, f, g, h, and i) mentioned inside the figures have been removed. 


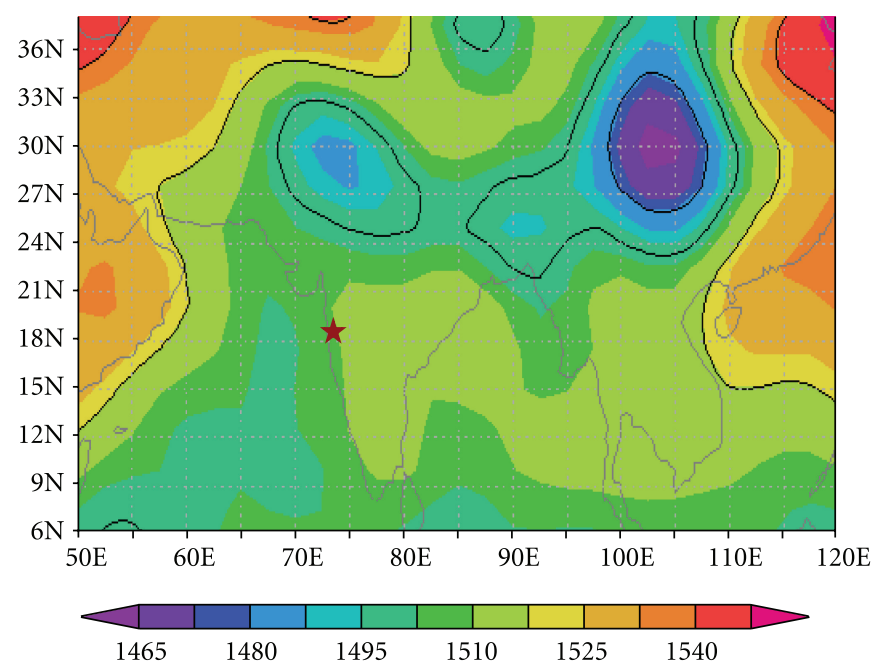

(a)

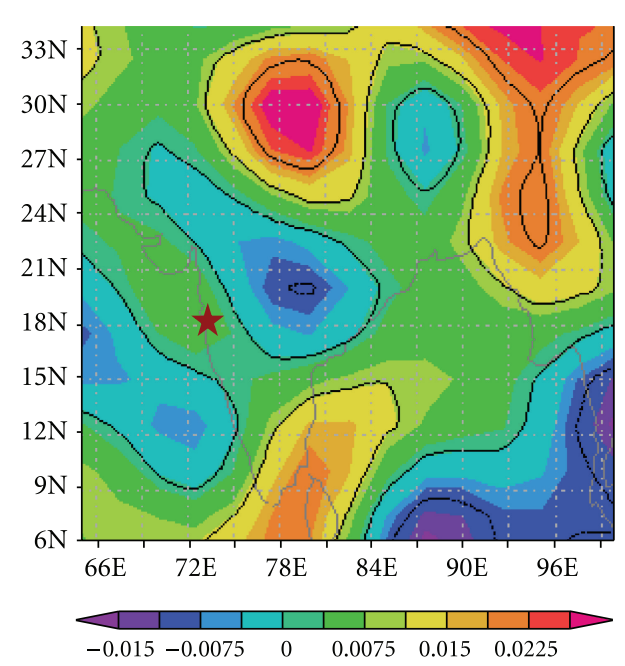

(b)

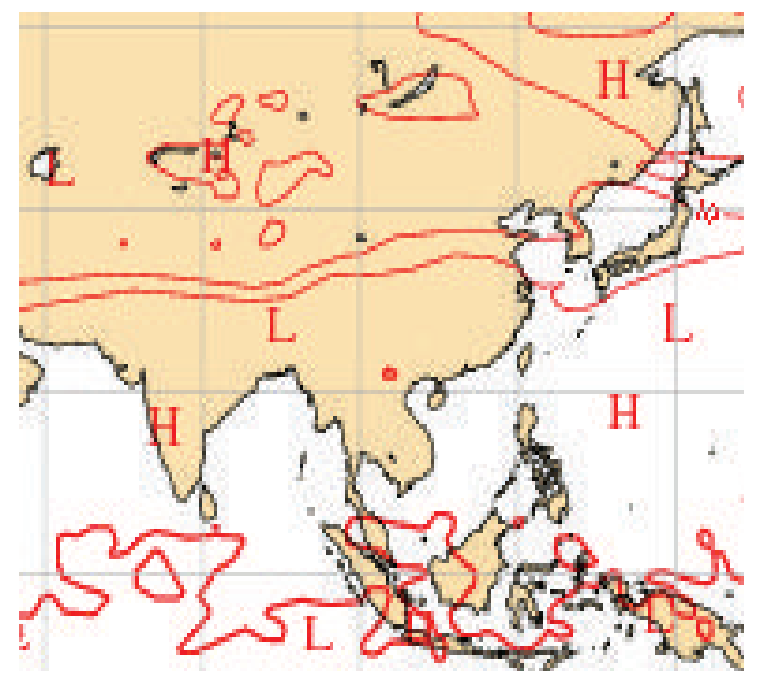

(d)

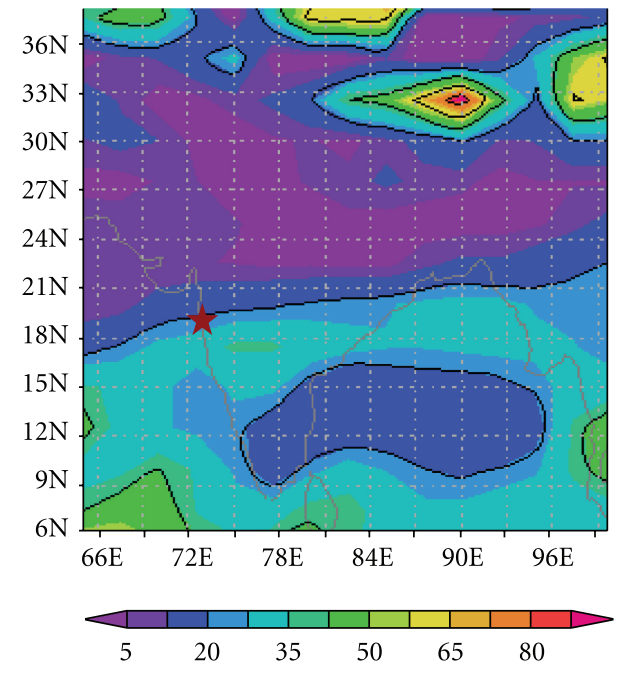

(c)

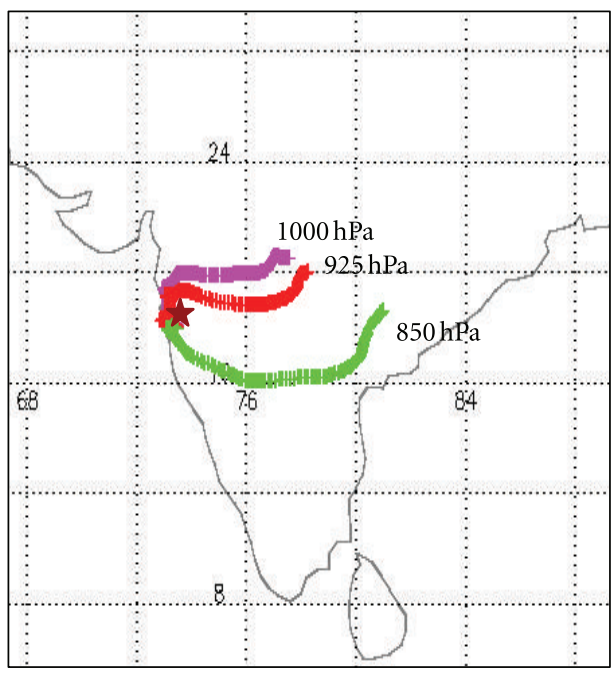

(e)

Figure 4: Continued. 


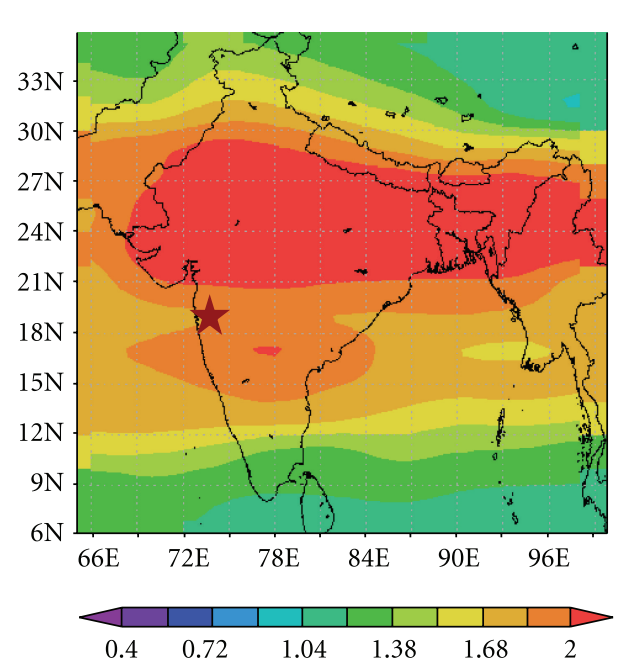

(f)

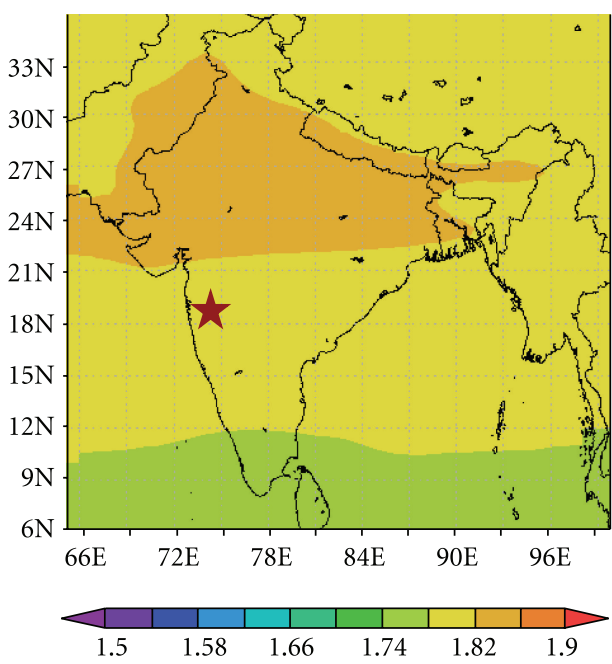

(g)

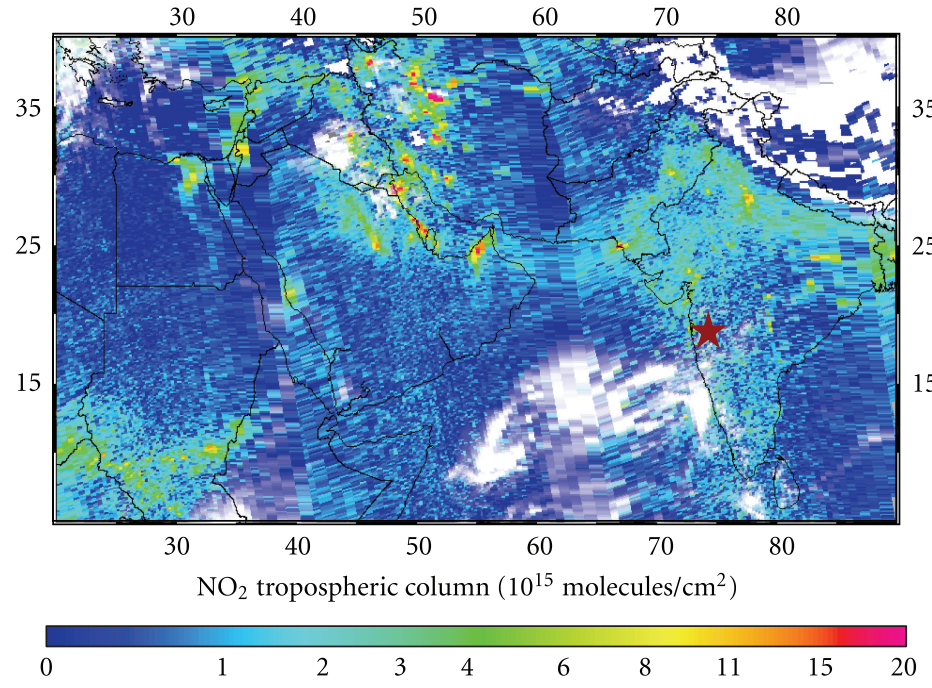

(h)

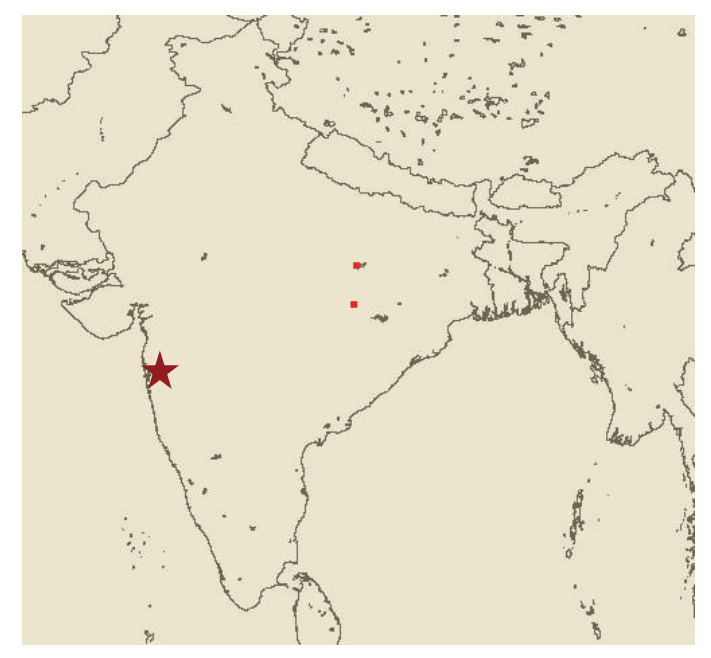

(i)

Figure 4: (a) Geopotential height (meter) map at $850 \mathrm{hPa}$, (b) vertical pressure velocity $\left(\mathrm{Pa} \cdot \mathrm{s}^{-1}\right)$ at the tropopause, (c) relative humidity $(\%)$ at $300 \mathrm{hPa}$ pressure level, (d) potential vorticity at the tropopause, (e) 5-day back trajectories at $1000 \mathrm{hPa}$ (pink), $925 \mathrm{hPa}(\mathrm{red})$, and $850 \mathrm{hPa}$ (green) pressure levels, (f) tropospheric CO $\left(10^{-1} \mathrm{ppmv}\right),(\mathrm{g}) \mathrm{CH}_{4}(\mathrm{ppmv}),(\mathrm{h}) \mathrm{NO}_{2}\left(10^{15} \mathrm{molecules} \cdot \mathrm{cm}^{2}\right)$, and (i) fire counts at Pune on 24 November 2006 (sources of data: NCEP Reanalysis, ECMWF, TES, OMI, and ASTR World Fire Atlas). 

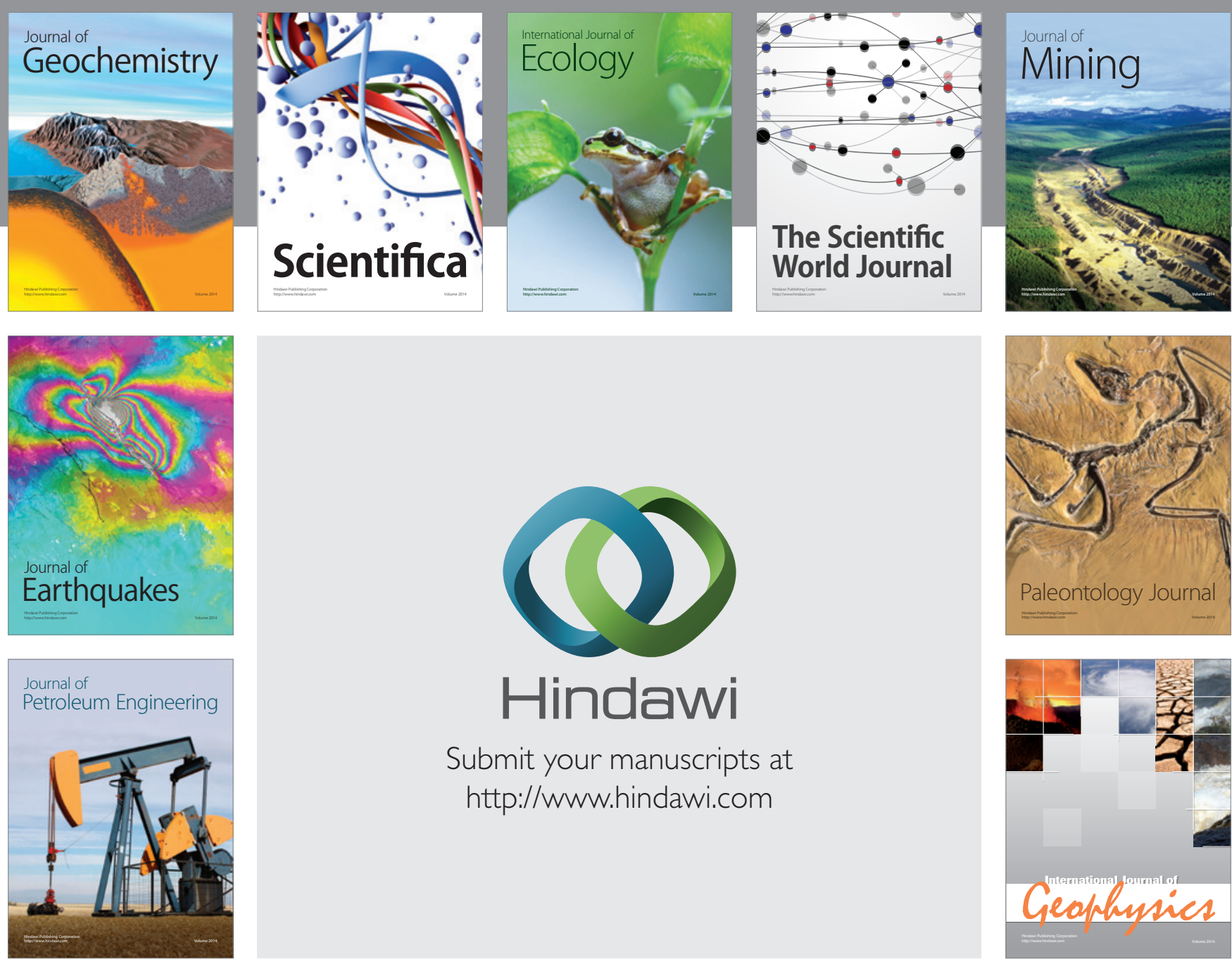

Submit your manuscripts at

http://www.hindawi.com
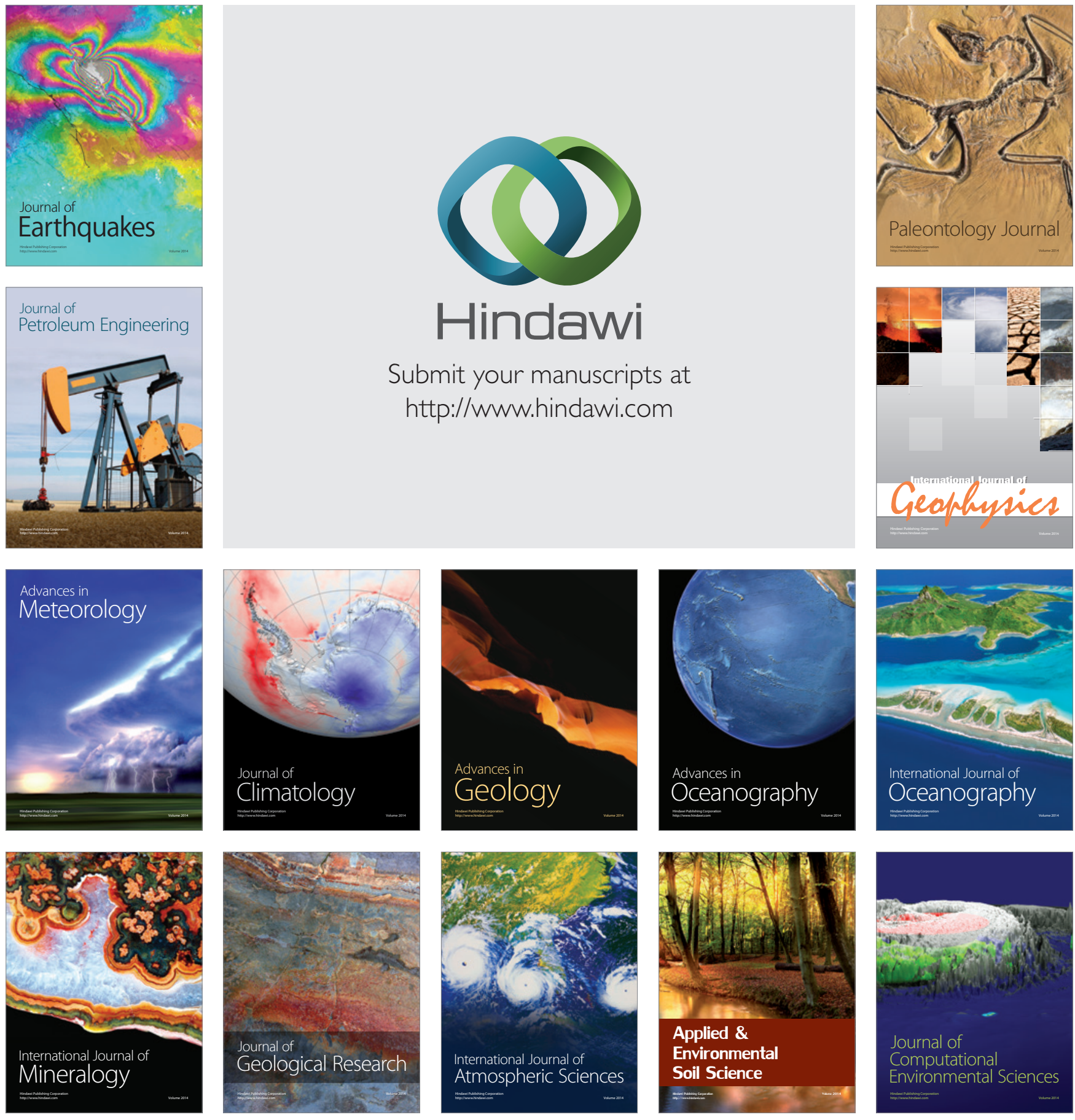